\title{
Answering Binary Causal Questions: A Transfer Learning Based Approach
}

\author{
Humayun Kayesh ${ }^{\dagger}$, Md. Saiful Islam ${ }^{\dagger}$, Junhu Wang ${ }^{\dagger}$, Shikha Anirban ${ }^{\dagger}$, A.S.M. Kayes ${ }^{\ddagger}$, Paul Watters \\ ${ }^{\dagger}$ School of Information and Communication Technology \\ Griffith University \\ Gold Coast, Australia \\ ${ }^{\ddagger}$ School of Engineering and Mathematical Sciences \\ La Trobe University \\ Melbourne, Australia \\ \{h.kayesh,saiful.islam,j.wang\}@griffith.edu.au, shikha.anirban@griffithuni.edu.au, \{a.kayes, p.watters $\} @ 1$ latrobe.edu.au
}

\begin{abstract}
Causal question answering is a task of answering causality related questions. The questions are referred to as binary causal questions when the questions e.g., "Could $X$ cause Y?" can be answered by yes/no answers. Answer to the previous question is yes if $X$ is a cause of $Y$, and otherwise no. The binary causal question answering systems can be used to validate causal relationships, which can be particularly useful for decision making. For example, it could be useful for the tourism authorities to know the answer to the question "Could growing social tension cause reduction in tourism?". We aim to automatically answer such binary causal questions by developing a machine learning model. However, training a machine learning model to detect causal relationships is challenging due to the lack of large and high quality labeled datasets. In this paper, we propose a transfer learning-based approach which fine-tunes pretrained transformer based language models on a small dataset of cause-effect pairs to detect causality and answer binary causal questions. The proposed approach achieves performance comparable to a number of benchmark approaches on five benchmark test datasets extracted by human experts conditioned on the same small training dataset.
\end{abstract}

\section{INTRODUCTION}

Binary causal questions ask whether there is a relationship between a candidate cause and a candidate effect. For example, in the following question "Could Australian bushfire cause a jump in carbon concentrations in the atmosphere?"1, "Australian bushfire" is a candidate cause and "a jump in carbon concentrations in the atmosphere" is a candidate effect. If there exists a causal relationship between the candidate cause and the candidate effect, the answer to the above binary causal question is either yes or no. The automatic answering of such binary casual questions might play an important role in everyday decision making and reasoning. It can be explored as an important tool by the decision makers to assess the situation and take informed decision. Additionally, a binary causal questions answering model may be used to discover new and previously unknown pairs of cause and effect as exemplified

\footnotetext{
1"The devastating bushfires in Australia are likely to cause a jump in carbon concentrations in the atmosphere this year, a forecast suggests, bringing the world closer to $1.5 \mathrm{C}$ of global heating." - https://www.theguardian.com/environment/2020/jan/24/australian-bushfireswill-cause-jump-in-co2-in-atmosphere-say-scientists
}

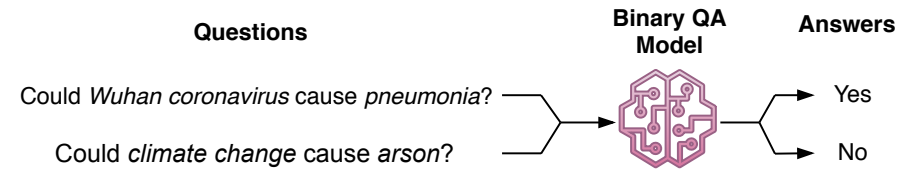

Fig. 1: An illustrative example of binary causal question answering (QA) model

and illustrated in Fig. 1 for "Could Wuhan coronavius cause pneumonia?"2 and "Could climate change cause arson?"3.

Answering causal questions is a trivial skill of human beings and often a human can answer causal questions without much effort as humans acquires knowledge about causality from the very beginning of their life through day-to-day life experiences and continuous learning. However, this trivial task for human is quite challenging for computers. To answer binary causal questions automatically, appropriate background knowledge (labeled training dataset) on causality is crucial, which is often hard to achieve. In the literature, there exists a number of approaches [1]-[11] to solve the automatic causality detection problem. The approaches apply various techniques such as linguistic rules [1], association rule mining [2], [3], causal network analysis [4], [5], and recently, application of neural network-based algorithms [6]-[11]. The rule-based approaches are often domain specific and not generalizable. The causal network-based approaches rely on exact word matching and neural network-based approaches require large training datasets. In general, the supervised machine learningbased approaches for causality detection require large and high quality labeled datasets to train. Unfortunately, high quality training data is often either expensive to acquire or not available. Also, when the training dataset becomes large, training machine learning models on such large datasets be-

\footnotetext{
2 "So far, we know the new Wuhan coronavirus causes pneumonia and therefore places an extra burden on hospitals."-http://theconversation.com/shouldwe-be-worried-about-the-new-wuhan-coronavirus-130366

3 "Victoria police say there is no evidence any of the devastating bushfires in the state were caused by arson."-https://www.theguardian.com/australianews/2020/jan/08/police-contradict-claims-spread-online-exaggeratingarsons-role-in-australian-bushfires
} 
comes computationally expensive. These drawbacks limit the applicability of such approaches. Hence, a machine learning approach that requires relatively small dataset to train, but still contains enough background knowledge on causality and achieves reasonably comparable performance is desirable.

In this paper, we aim to answer binary causal questions by applying transfer learning based approach as it allows us to utilize existing generalized background knowledge for our task specific needs by fine-tuning the model with a relatively small training dataset. We apply pretrained transformer based language models such as Bidirectional Encoder Representation from Transformers (BERT) and its variants to exploit the already learned model weights to solve the problem of large dataset requirements. In particular, we found that the WordPiece tokenization [12] technique used in BERT models is effective to deal with the exact word matching problem in our task. We also proposed an approach to automatically extract a training dataset consisting of cause and effect pairs from news articles by applying causal cue words and then use this dataset to fine-tune the models for our binary causal question answering task. We list our contributions as given as below:

1) we propose a transfer learning-based approach that applies BERT language models to answer binary causal questions;

2) we also propose an automatic approach to collect quality training dataset from news articles to fine-tune the BERT models to detect causality; and

3) we perform extensive experiments to show the effectiveness of the proposed approach.

The rest of the paper is organized as follows. Section II reviews the existing state-of-the-art approaches. Section III presents our approach. Section IV discusses the experimental results. Finally, Section V concludes the paper.

\section{RELATED WORK}

Causality detection from text and question answering are both active research areas. The causality detection approaches that are relevant to our problem includes linguistic rules-based approach, graph-based approach, machine learning-based approach and very recently neural network-based approach.

A rule-based approach on causality detection is proposed by Radinsky et al. [1] that automatically generates a set of rules that are applied to detect causal relationship. However, the rules are dependent on the grammatical correctness of sentences, which is not always available or sometimes missing in open texts. Background knowledge is often useful to detect causality. The graph-based approaches [4], [5] encode background knowledge in causal network and use that knowledge to detect causality. Luo et al. [4] propose a graph-based approach that collects a set of causal sentences from web using a set of causal cue words. Sentences are then split into causal phrases and effect phrases. A Cartesian product between the words in a causal phrase and the corresponding effect phrase is taken to prepare a list of word pairs. The first word in the pair comes from the causal phrase and the other word comes from the effect phrase. The pairs are then used to build a directed causal graph. The nodes in the graph correspond to the words and the edges represent a causal relation. Each edge contains a weight. For example, a directed link from node A to node $\mathrm{B}$ represents the number of times the word $\mathrm{A}$ were used as a cause and B as an effect. These values are then used to calculate the combined causal scores of candidate cause-effect pairs. However, this approach cannot capture the multi-word expression as it tokenizes every word in a phrase [5]. Also, this approach cannot detect causality if either of the words on a pair is missing in the causal graph.

A causal questions answering method is proposed in [8] that aims to rerank answers to the causal questions using embeddings. This approach collects causal pairs from free text and then converts them into causal embeddings. Both causal and effect embeddings are then passed to two separate Convolutional Neural Networks (CNN). The outcomes of the CNNs are Max Pooled and then merged together by calculating cosine similarity. The Softplus activation function is used in the final layer with a single node to detect causality. Since this approach is trained solely on the training data, it requires a large training data for training. Dasgupta et al. [6] propose a neural networkbased approach to extract cause, effect and causal connectives in a sentences. The approach uses both word features and linguistic features to train a Bidirectional Long Short-Term Memory (Bi-LSTM) model to detect causality. The input to the model is a combination of word vectors and linguistic vectors. The authors used a pretrained GloVe model to convert each word into a 300-dimensional vector. The linguistic features are composed of both syntactic features and semantic featuers. The syntactic featuers include part-of-speech tags, dependency relationship, and positions of noun and verbs. The semantic features includes nine noun hierarchy in WordNet proposed in [13] along with the grammatical structure of the sentence. Finally, the trained model is used to label each word whether the word is a cause, an effect, a causal connective or none. However, none of the above approaches targets automatic binary causal question answering task.

To the best of our knowledge, there exists one work on binary causal question answering task and the approach is called Natural Language Model - Bidirectional Encoder Representation from Transformers (NLM-BERT), which is proposed by Hassanzadeh et al. [7]. The authors assume that the relationship between cause and effect is unidirectional. More specifically, if $(\mathrm{X}, \mathrm{Y})$ is a causal pairs and " $\mathrm{X}$ may cause $Y$ " is true, then it is less likely that "Y may cause $X$ " will be true. The authors collected a corpus of 17 million (17M) causal sentences and converted them into vectors using a pretrained BERT model. For a candidate $(X, Y)$ pairs, it finds 10 closest sentences ( $k$-nearest neighbor approach) for both " $\mathrm{X}$ may cause $Y$ " and "Y may cause X". Then it calculates the average cosine similarity for both of the cases. Two threshold values are used to finally derive the yes/no answer for "Could $\mathrm{X}$ cause Y?". The main drawback of this approach is that the threshold values are not previously known and varies from test dataset to another test dataset. Also, it requires expert 
TABLE I: List of causal cue words used to prepare the training dataset (adapted from [9], [10])

\begin{tabular}{|c|c|c|c|c|c|c|c|}
\hline affect & because & causes & due to & if & induce & owing to & results from \\
\hline \begin{tabular}{|l} 
affected by \\
\end{tabular} & because of & causing & effect of & if..., then & induced & reason for & so that \\
\hline and consequently & brings on & $\mathrm{cOZ}$ & gave rise to & in response to & lead to & reasons for & the result is \\
\hline as a consequence & cause & decrease & given rise to & increase & leads to & result from & therefor \\
\hline as a consequence of & caused & decreased by & giving rise to & increased by & led to & resulted from & thus \\
\hline as a result of & caused by & decreases & hence & increases & on account of & resulting from & \\
\hline
\end{tabular}

knowledge to set the correct threshold values.

In this paper, we propose a transfer learning-based approach to deal with the above mentioned challenges of automatic answering binary causal questions. We propose to use a pretrained BERT model similar to Hassanzadeh et al. [7] that comes with a rich linguistic information. Unlike the approach proposed by Hassanzadeh et al. that applies $k$ nearest neighbour-based cosine similarity calculation on large dataset, we fine-tune the BERT model with a relatively small training dataset to contextualize the model for the binary causal question answering task. In our approach, we also avoid the manual threshold selection for the test datasets - threshold selection is not dependent on test dataset. Once our model is fine-tuned on the training dataset, it can be applied for each of the test datasets without the need of selecting any dataset specific thresholds.

\section{OUR APPROACH}

In this section, we describe our transfer learning-based approach to answer binary causal questions. At first, we extract and prepare a training dataset from news articles and then we fine-tune BERT models on this training dataset. The fine-tuned BERT model is then used to answer binary causal questions.

\section{A. Extraction of Training Data}

We apply a semi-supervised approach to prepare the training dataset. In our approach, we extract a set of causal and noncausal pairs from one million news articles [14]. To get the causal pairs, we extract the sentences that contain at least one causal cue words e.g., causes, due to, and because of (see the complete list as given in Table I). Then we extract causal pairs such as $(X, Y)$ from the sentences using those causal cue words where $X$ corresponds to the causal phrase and $Y$ corresponds to the effect phrase. In our training dataset, we label each of these pairs as causal. We also extract noncausal training data from the same news articles dataset. To avoid any data overlap with the causal pairs, we extract the sentences that do not contain any causal cue words. We divide each sentence into half to prepare the non-causal pairs $(X, Y)$, where $\mathrm{X}$ represents the first half and and $\mathrm{Y}$ represents the second half of the sentence. Each of these pairs of data is labeled as not_causal as we assume that these sentences should not contain any causality.

Table II illustrates the training dataset statistics which includes the total number of pairs, vocabulary size and the size of the phrases. One prominent characteristic that is visible
TABLE II: Training dataset statistics

\begin{tabular}{l|l|l}
\hline \multicolumn{1}{c|}{ Name } & Causal & Non_causal \\
\hline \hline Number of pairs & 100000 & 100000 \\
\hline Vocabulary size & 27206 & 63471 \\
\hline Longest phrase size & 55 & 189 \\
\hline Shortest phrase size & 1 & 2 \\
\hline Average phrase size & 3.5 & 9.23 \\
\hline
\end{tabular}

from the table is that the non-causal pairs in the training dataset are around 3 times longer than the causal pairs. Also, the non-causal pairs contains more unique vocabulary than the causal pairs, i.e., there are 27206 and 63474 unique words in the causal and non-causal pairs, respectively. This is because, we applied linguistic rules such as " $\mathrm{X}$ causes $\mathrm{Y}$ " and " $Y$ because of $X$ ", to extract the causal phrase $X$ and the effect phrase $Y$ from the sentence while discarding other non-relevant words. However, the full sentences are split into halves without removing any word to prepare the non-causal pairs $(X, Y)$ so that both $X$ and $Y$ contain linguistic flows. Fig. 2 illustrates a few lexical frequencies of the causal phrases in the training dataset. We notice from Fig. 2(a) that the most frequent five causal cue words are if, because, cause, due to, and because of. Fig. 2(b) and Fig. 2(c) display the top causal bigrams and effect bigrams, respectively. The causal bigrams are extracted from the causal phrases and similarly, the effect bigrams are extracted from the effect phrases. Intuitively, the frequent causal bigrams such as risk uncertainty, new information and uncertainty factors are part of causes in the causal relationships. Similarly, the frequent effect bigrams such as actual results, differ materially and result differ are the part of effects in the causal relationships. Fig. 3 and Fig. 4 illustrate the word co-occurrence heatmaps of the causal phrases and the effect phrases respectively, which also support the bigrams figures described above. We find that the dataset prepared by following the above approach is good enough to train a transfer learning model to achieve a comparable performance to the state-of-the-art approaches.

\section{B. Pair-to-Sentence Conversion}

After collecting the training dataset (refer to Section III-A) that contains both causal and non-causal pairs, we fine-tune a pretrained BERT model (pretrained on large dataset to capture general linguistic features) on this dataset. To do so, we first convert each causal pairs $(X, Y)$ into a sentence by following the Hassanzadeh et al. [7]'s technique and represent the pair as "X may cause Y". For example, the pair (Australian 


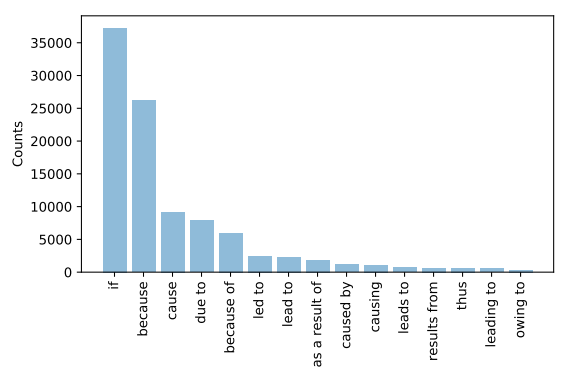

(a) Causal cue words

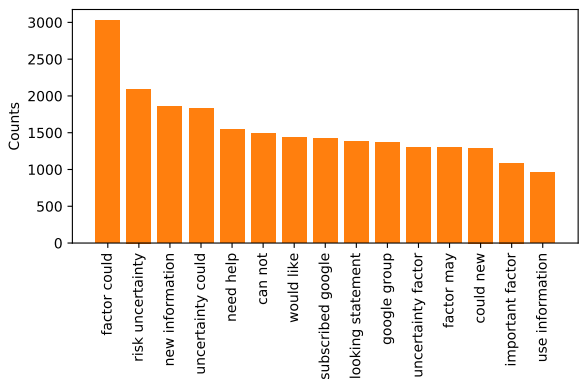

(b) Causal bigrams

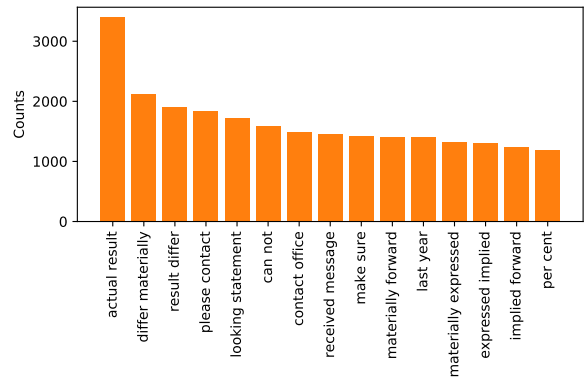

(c) Effect bigrams

Fig. 2: Top 15 causal cue words, causal and effect bigrams

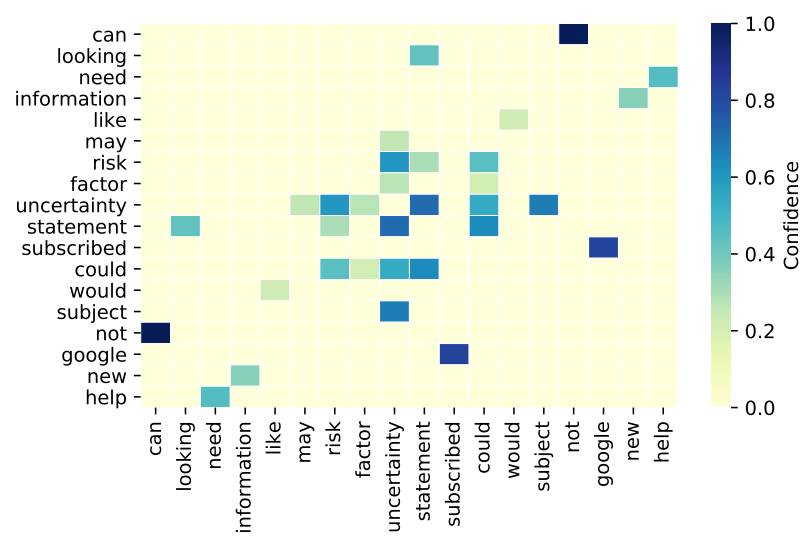

Fig. 3: Heatmap of causal words

fire, a jump in carbon concentrations in the atmosphere) is represented as "Australian fire may cause a jump in carbon concentrations in the atmosphere".

\section{Fine-tuning BERT Model}

BERT [15] is a powerful transformer-based language model. Recently, it has been used to build the state-of-the-art models for at least eleven natural language processing tasks. The BERT model is trained on a large dataset of BooKCorpus [16] (16GB) and English Wikipedia text. A pretrained BERT can capture the bidirectional representation of a sentence as it applies a deep bidirectional encoder mechanism. The power of BERT relies on the captured language representation during the pretraining phase. Though BERT model is trained for generic tasks such as context word and next sequence prediction, the model can also be fine-tuned to transfer its rich linguistic knowledge for more task specific needs. We believe that the rich linguistic knowledge patterns captured by BERT can be utilized to detect causality too. Hence, we apply a pretrained BERT model to answer binary causal questions by fine-tuning the model on our training dataset.

Before we perform the fine-tuning of the BERT model, each of the training pairs are converted into a sequence such as " $\mathrm{X}$ may cause Y" as described in Section III-B. The sequence is then passed to the BERT tokenizer which preprocesses the sentence into a format that is compatible to the BERT model. More specifically, the BERT tokenizer tokenizes each sentence into wordpieces [12] and each wordpiece is encoded with their

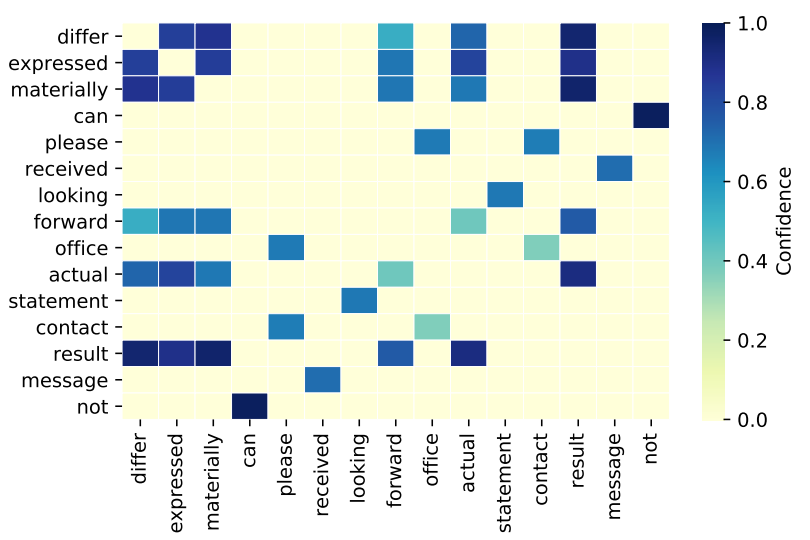

Fig. 4: Heatmap of effect words

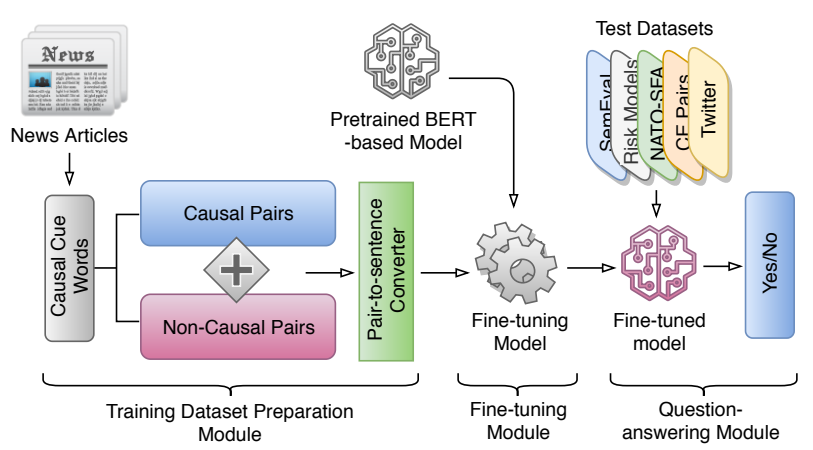

Fig. 5: Transfer learning based binary causal QA work flow

corresponding dictionary indexes. The word dictionary is also prebuilt during the pretraining phase. The encoded wordpiece sequences are then passed to the BERT model for fine-tuning. Once the fine-tuning is done, the model is then used on the test datasets for evaluation.

The overall workflow of our proposed BERT-based transfer learning approach for binary causal question answering framework is depicted in Fig. 5.

\section{EXPERIMENTS}

In this section, we describe our evaluation datasets and their source and attributes. We also discuss the benchmark approaches such as CausalNet approach [4] and NLM-BERT [7] that we have implemented to compare with our approach. The NLM-BERT model [7] was based on 17 million causal 
TABLE III: Sample items from the SemEval dataset

\begin{tabular}{l|l|l|l}
\hline \multicolumn{2}{c|}{ Causal } & \multicolumn{2}{c}{ Non_causal } \\
\hline \hline $\mathbf{X}$ & $\mathbf{Y}$ & $\mathbf{X}$ & $\mathbf{Y}$ \\
\hline generator & energy & protein & researchers \\
\hline lamp & light & rocks & pile \\
\hline collision & fire & copper & tissue \\
\hline attack & sorrow & article & criticisms \\
\hline unemployment & alcoholism & drum & ear \\
\hline gun & beam & work & difficulties \\
\hline
\end{tabular}

TABLE IV: Sample items from the NATO-SFA dataset

\begin{tabular}{l|l|l}
\hline $\mathbf{X}$ & $\mathbf{Y}$ & Label \\
\hline \hline $\begin{array}{l}\text { currency appreciation } \\
\text { against US dollar }\end{array}$ & lower demand for USD & causal \\
\hline country in state of war & fair justice system & non_causal \\
\hline $\begin{array}{l}\text { Technological } \\
\text { dependencies }\end{array}$ & Vulnerabilities & causal \\
\hline Fair burden sharing & $\begin{array}{l}\text { increased cost of doing } \\
\text { business }\end{array}$ & causal \\
\hline $\begin{array}{l}\text { weakening economic envi- } \\
\text { ronment }\end{array}$ & $\begin{array}{l}\text { foreign data providers dom- } \\
\text { inate market }\end{array}$ & non_causal \\
\hline Climate Change & New opportunities & causal \\
\hline
\end{tabular}

sentences and the two thresholds of the model are test dataset dependent as we discuss in Section II. In this paper, we improve the NLM-BERT model by proposing an approach for threshold selection that is not test dataset dependent, but can be learned from the training dataset. We have also implemented CausalNet approach [4] based on our own training dataset.

\section{A. Evaluation Datasets}

We evaluate our approach on five benchmark datasets four datasets published by [7] and a Twitter dataset from our previous work [9]. Each dataset consists of pairs of texts such as $(X, Y)$ and the corresponding labels. A brief description of these datasets is given below:

- SemEval - The SemEval dataset is a subset of the SemEval 2010 task 8 [17] dataset. The dataset is about identifying relationship between words in word pairs. Our SemEval dataset contains 1730 pairs which includes 865 causal and 865 non_causal pairs. The causal pairs are taken directly from the original dataset. The non_causal pairs are prepared by collecting pairs of words that has no causal relationship between them. Table III displays a few samples from the dataset. Any pairs in the table can be converted into a binary causal question. For instance, (collision, fire) is an example pair and "Could collision cause fire?" is the corresponding binary causal question.

- NATO-SFA - The NATO-SFA dataset is prepared from the Strategic Foresight Analysis (SFA) 2017 report [18] published by NATO (the North Atlantic Treaty Organization). The report includes a set of "trend" of changes in the world and their "implications" curated by human experts. The trends are considered as causes and the corresponding implications are considered as the effects. In the NATO-SFA dataset we have 118 pairs of words or phrases in total. There are 59 causal pairs and 59 non_causal pairs. The non_causal pairs are prepared by
TABLE V: Sample items from the Risk Models dataset

\begin{tabular}{l|l|l}
\hline $\mathbf{X}$ & $\mathbf{Y}$ & Label \\
\hline \hline Rising regional tensions & resource competition & non_causal \\
\hline decrease in taxes & market disruptors & causal \\
\hline growing social tension & reduced tourism & causal \\
\hline increase in taxes & $\begin{array}{l}\text { liberal politician or party } \\
\text { elected }\end{array}$ & causal \\
\hline $\begin{array}{l}\text { strengthening economic } \\
\text { environment }\end{array}$ & $\begin{array}{l}\text { increased cost of doing } \\
\text { business }\end{array}$ & non_causal \\
\hline Natural disasters & $\begin{array}{l}\text { Increased requirement for } \\
\text { humanitarian support }\end{array}$ & causal \\
\hline
\end{tabular}

TABLE VI: Sample items from the CE pairs dataset

\begin{tabular}{l|l|l}
\hline $\mathbf{X}$ & $\mathbf{Y}$ & Label \\
\hline \hline broadband access & more new businesses & causal \\
\hline increased growth & $\begin{array}{l}\text { dent consumer and business } \\
\text { confidence }\end{array}$ & non_causal \\
\hline $\begin{array}{l}\text { consistent branding and } \\
\text { pricing }\end{array}$ & increases revenue & causal \\
\hline increase in corruption & $\begin{array}{l}\text { negative effect on foreign } \\
\text { investment }\end{array}$ & causal \\
\hline management incentives & low investment & causal \\
\hline $\begin{array}{l}\text { increased government } \\
\text { spending }\end{array}$ & decreased political stability & non_causal \\
\hline
\end{tabular}

combining two phrases or words that has no direct causeeffect relationship in the report. Table IV shows a set of example pairs for the NATO-SFA dataset.

- Risk Models - The Risk Models dataset is built using a set of models that is a part of a decision support system [19], [20]. Each model is a graph where the nodes represent an event and the edges represent a cause-effect relationship between two nodes. The dataset contains 402 causal pairs which are prepared from the relationships between edges and nodes. The dataset also has the same number of non_causal pairs that are prepared by combining unrelated edges and nodes. Table V illustrates a few sample pairs from the Risk Models dataset.

- CE Pairs - The CE (cause-effect) pairs dataset is an extension of the Risk Models dataset. To build the CE pairs dataset a set of node labels is assigned to 7 human annotators and asked to find corresponding cause or effect phrases by web search. The node labels of the model graphs are used as the cause or effect phrases and the corresponding cause of effect phrases are searched on the web. The dataset contains 302 pairs, where $50 \%$ of the pairs are causal and the remaining pairs are non_causal. Table VI shows a few example pairs from the dataset.

- Twitter - This dataset is prepared by Kayesh et al. [9] that contains labeled tweets on causality. The tweets in the dataset are related to Commonwealth Games, Gold Coast 2018. The dataset includes total 916 pairs with manually annotated labels. Table VII includes a set of example causal and non_causal pairs from this dataset.

\section{B. Benchmark Approaches}

We compare our approach to answer binary causal questions with a number of existing benchmark approaches. A brief description of the benchmark approaches is given below. 
TABLE VII: Sample items from the Twitter dataset

\begin{tabular}{l|l|l}
\hline \multicolumn{1}{c|}{$\mathbf{X}$} & \multicolumn{1}{c|}{ Y } & \multicolumn{1}{c}{ Label } \\
\hline \hline i ned to be front and centre & it's al about me & non_causal \\
\hline $\begin{array}{l}\text { families truly suport girl- } \\
\text { child }\end{array}$ & $\begin{array}{l}\text { we can se that sky to is not } \\
\text { the limit }\end{array}$ & causal \\
\hline $\begin{array}{l}\text { you're loking for us in the } \\
\text { vilage }\end{array}$ & you'l know where to find us & non_causal \\
\hline $\begin{array}{l}\text { \#comonwealthgames2018 } \\
\text { and what did they do }\end{array}$ & $\begin{array}{l}\text { people were urged to stay } \\
\text { out of gold coast }\end{array}$ & non_causal \\
\hline they are the best & both reached this point & causal \\
\hline a mechanical isue & $\begin{array}{l}\text { 34am central to benleigh } \\
\text { train has ben canceled }\end{array}$ & casual \\
\hline
\end{tabular}

1) NLM-BERT Model and its Variants: This section presents the NLM-BERT Model proposed by Hassanzadeh et al. [7] and its variants that we have implemented in our paper.

- NLM-BERT-17M [7] - This is the BERT-based natural language model proposed by Hassanzadeh et al. [7]. The approach uses $17 \mathrm{M}$ causal sentences as their training dataset. Since we couldn't access their training dataset to reproduce the results we directly report the same thresholds and results mentioned in the original paper [7] on four test datasets: SemEval, NATO-SFA, Risk Models and CE Pairs. The similarity calculation of NLM-BERT and threshold selection are described later in this section.

- NLM-BERT [7] - To implement NLM-BERT that is local to our training dataset, we use the pretrained 'SentenceTransformer' [21] model to transform each sentence into a 768-dimension vector. For indexing the sentence vectors and searching the closest $k$ sentences, we use the faiss ${ }^{4}$ library [22] as suggested by the authors [7]. For this model, we also use the same thresholds proposed by Hassanzadeh et al. [7] for all test datasets except the twitter dataset. For the twitter dataset, we have used the maximum of the thresholds proposed by Hassanzadeh et al. [7] for SemEval, NATO-SFA, Risk Models and CE Pairs.

- NLM-BERT++ - The implementation of this model is the same as the NLM-BERT model [7] except the usage of thresholds. Unlike the NLM-BERT model, we use a single pair of values for $t h_{1}$ and $t h_{2}$ which is 0.60 and 0.30 , respectively, for all test datasets. These threshold values are determined automatically by following a technique described later in this section.

**NLM-BERT Similarity Scores Calculation**. The NLM-BERT model [7] is dependent on two similarity scores: bert-sim-score and bert-c-score. To calculate these scores, the model converts a candidate causal pair $(\mathrm{X}, \mathrm{Y})$ into two sentences "X may cause $Y$ " and "Y may cause $X$ ". Then, it calculates bert-sim-score and bert-c-score for " $\mathrm{X}$ may cause Y" and "Y may cause X", respectively. To calculate bert-simscore, the approach converts the sentence " $\mathrm{X}$ may cause $\mathrm{Y}$ " into a vector $v_{f}$ using a pretrained BERT model and then, finds a set of $k$ closest vectors $\left\{v_{0}, v_{1}, \ldots, v_{k-1}\right\}$ from the training dataset which has been converted into a list of vectors using

\footnotetext{
${ }^{4}$ https://github.com/facebookresearch/faiss
}

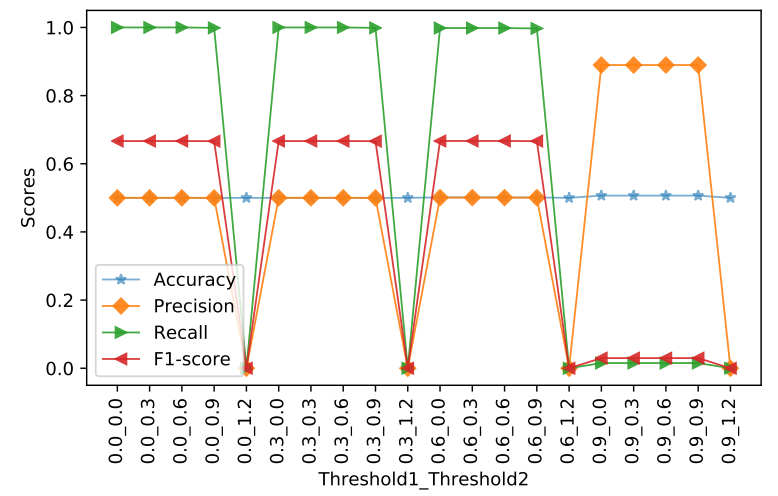

Fig. 6: Threshold selection for the NLM-BERT++ approach

the same pretrained BERT model. Then, it calculates bertsim-score based on the average cosine similarity of the pairs $\left(v_{f}, v_{i}\right)$, where $i \in\{0,1, \ldots, k-1\}$ as given in Eq. 1 .

$$
\text { bert-sim-score }=\frac{\sum_{i=0}^{k-1} \operatorname{CosineSimilarity}\left(v_{f}, v_{i}\right)}{k}
$$

To calculate bert-c-score, the reverse causal sentence "Y may cause X" is similarly converted into a vector $v_{r}$ and the closest $k$ sentence vectors $\left\{v_{0}, v_{1}, \ldots, v_{k-1}\right\}$ are extracted from the training dataset. The average cosine similarity score, which is labeled as bert-reverse-sim-score, is calculated based on the pairs $\left(v_{r}, v_{j}\right)$, where $j \in\{0,1, \ldots, k-1\}$ as given in Eq. 2 . Finally, bert-c-score is calculated by dividing bert-sim-score by bert-reverse-sim-score as given in Eq. 3 .

$$
\begin{aligned}
\text { bert-reverse-sim-score } & =\frac{\sum_{j=0}^{k-1} \operatorname{CosineSimilarity~}\left(v_{r}, v_{j}\right)}{k} \\
\text { bert-c-score } & =\frac{\text { bert-sim-score }}{\text { bert-reverse-sim-score }}
\end{aligned}
$$

**Automatic Threshold Selection for NLM-BERT++**. In NLM-BERT model [7], the answer to the causal question "Could X cause Y?" depends on bert-sim-score and bert$c$-score, which are maximized by thresholds $t h_{1}$ and $t h_{2}$, respectively. If bert-sim-score and bert-c-score is greater than $t h_{1}$ and $t h_{2}$, respectively, then the answer is yes and otherwise, the answer is no as given in Eq. 4.

$$
f(X, Y)= \begin{cases}y e s & \text { if bert-sim-score }>t h_{1} \& \text { bert-c-score }>t h_{2}, \\ n o & \text { otherwise }\end{cases}
$$

In the original NLM-BERT approach [7], $t h_{1}$ and $t h_{2}$ need to be finalised for each test dataset, which requires expert supervision. For this, a prior knowledge of the test dataset characteristics is required. In this paper, we aim to eliminate this manual threshold selection process by automatically learning the thresholds from the training data (not the test dataset). We randomly split the training dataset into training and validation datasets. We use $75 \%$ data for training and the remaining $25 \%$ data for validation. Then, we follow the 


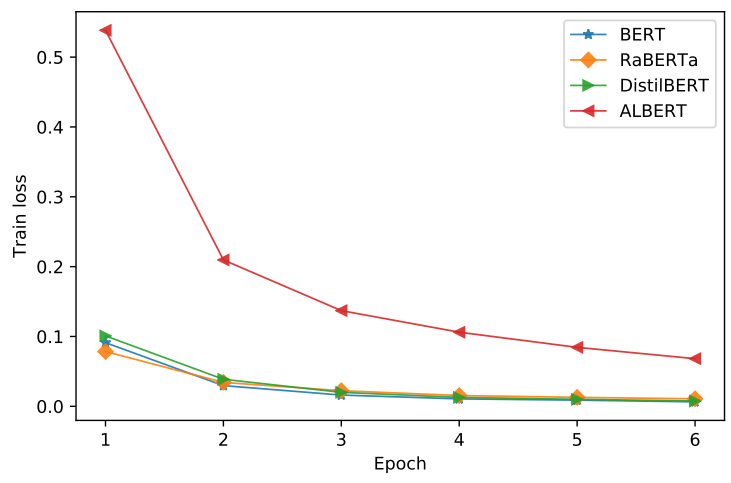

(a) Training Loss

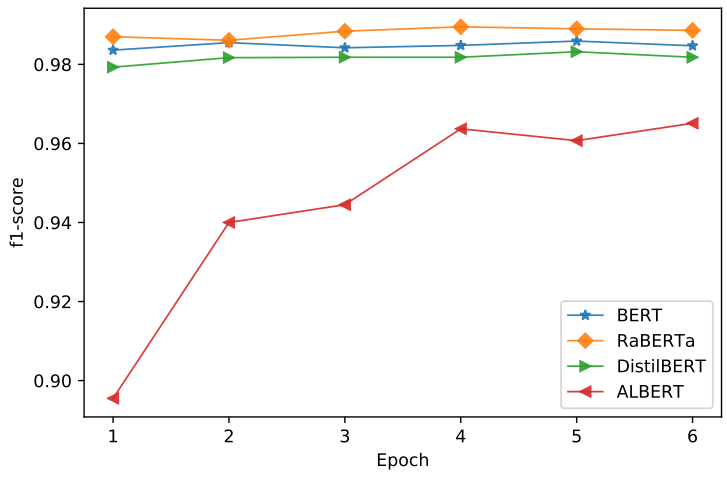

(b) Validation F1-score

Fig. 7: Pretraiend BERT-based models fine-tuning scores on the validation dataset

same procedure described in NLM-BERT Scores Calculation to calculate the two scores and use Eq. 4 to answer binary causal questions and evaluate them on the validation dataset for different combination of $t h_{1}$ and $t h_{2}$. For $t h_{1}$, the value varies from 0.0 to 1.0 and the threshold $t h_{2}$ ranges between 0.0 and 1.2. We set the step size to 0.3 that is used to increase these threshold values. Fig. 6 illustrates the validation scores for different combinations of thresholds. We observe that both f1-score and accuracy are maximized for $t h_{1}=0.6$ and $t h_{2}=0.3$ as it is evident from Fig. 6 .

2) CausalNet Approach: The CausalNet approach [4] builds a causal network, which is a bidirectional graph where each node represents a word and each edge corresponds to a causal relationship, to capture the causal knowledge. Firstly, the training dataset is preprocessed using the set of causal cue words given in Table I. For a candidate causal pairs $(X, Y)$, a causal score is calculated using the causal graph to decide whether X is a cause of Y. Firstly, a set of word pairs is prepared by implementing Cartesian product between words in both $X$ and $Y$. For each pair, the necessity and sufficiency causal scores are calculated from the causal network, which are then combined together after maximizing by a threshold. Finally, the pair-wise scores are summed together and divided by the total number of words in $\mathrm{X}$ and $\mathrm{Y}$ to calculate the causal score. Interested readers are referred to [4] for detail explanation of the approach. In this experiment, we use the same $100 \mathrm{~K}$ training causal pairs to build the causal network and considered a candidate pair (X, Y) as causal if the causal score is greater than zero.

3) Our Approach: This section presents the automatic binary question answering models that we have implemented based on the state-of-the-art BERT model and its variants by following the approach described in Section III.

- BERT [15] - BERT is a transformer-based language model proposed by Google Research. This model can be used for transfer learning based natural language processing (NLP) tasks. To implement this BERT model based transfer learning for our task, we use the transformers ${ }^{5}$ library [23], which provides several pretrained

\footnotetext{
${ }^{5}$ https://github.com/huggingface/transformers
}

transformers based language models. In our experiment, we use the 'bert-base-uncased' version of the pretrained model with 'BertForSequenceClassification' class.

- RoBERTa [24] - RoBERTa is a variation of the BERT model, which is proposed by Facebook AI. This approach focuses on hyperparameter tuning and alternative training strategies of BERT. The authors claim state-of-the-art performance on at least three publicly avaiable datsets. To implement this model in our experiment, we use the 'roberta-base' version of the pretrained model, which is released by the transformers library [23] with the 'RobertaForSequenceClassification' class.

- DistilBERT [25] - DistilBERT is a light-weight variation of BERT. The objective of this model is to reduce the number of hyper parameters and structural complexity of transformers while keeping the performance comparable to the original BERT model. The version of pretrained model we implement in this experiment is 'distilbertbase-uncased' and we use the "DistilBertForSequenceClassification" class to fine-tune the model.

- ALBERT [26] - ALBERT is another light-weight version of the BERT model. The ALBERT model is proposed to handle the issue of large training time, higher usage of memory and scaling for the large dataset. The approach applies parameter reduction technique to reduce run-time and improve memory usage. In this experiment, we finetune the 'albert-base-v2' version of the pretrained model with 'AlbertForSequenceClassification' class.

\section{Experiment Settings}

We perform the experiments in this paper on Google Colab GPU runtime, which offers $12 \mathrm{~GB}$ of memory and a Tesla K80 GPU processor that has 2,496 CUDA cores. We run every BERT-based model by setting batch size to 32 , maximum sequence length to 128 , and learning rate to 0.00002 . We use a small learning rate so that the pretrained weights are not overridden too much. Fig. 7 suggests that the training losses and validation f1-scores of BERT, RoBERTa and DistilBERT are plateau at around epoch 4 or 5. From these observations, we set the number of epochs to 5 for BERT, 4 for RoBERTa, 
TABLE VIII: Pretrained BERT-based models fine-tuning scores

\begin{tabular}{c|l|l|l|l|l|l|l}
\hline Model & Score & Epoch 1 & Epoch 2 & Epoch 3 & Epoch 4 & Epoch 5 & Epoch 6 \\
\hline \hline \multirow{3}{*}{ BERT } & Train loss & 0.0911 & 0.0296 & 0.0161 & 0.0106 & 0.0088 & 0.0064 \\
\cline { 2 - 8 } & Val Acc & 0.9842 & 0.986 & 0.9847 & 0.9855 & 0.9864 & 0.9853 \\
\cline { 2 - 8 } & Val F1 & 0.9836 & 0.9855 & 0.9842 & 0.9848 & $\mathbf{0 . 9 8 5 9}$ & 0.9847 \\
\hline \hline \multirow{3}{*}{ RoBERTa } & Train loss & 0.0784 & 0.0342 & 0.0223 & 0.0154 & 0.0128 & 0.0107 \\
\cline { 2 - 8 } & Val Acc & 0.9876 & 0.9866 & 0.9888 & 0.9898 & 0.9893 & 0.989 \\
\cline { 2 - 8 } & Val F1 & 0.987 & 0.9861 & 0.9884 & $\mathbf{0 . 9 8 9 5}$ & 0.989 & 0.9886 \\
\hline \hline \multirow{3}{*}{ DistilBERT } & Train loss & 0.1008 & 0.0387 & 0.02 & 0.0127 & 0.01 & 0.0076 \\
\cline { 2 - 8 } & Val Acc & 0.9799 & 0.9825 & 0.9823 & 0.9823 & 0.9839 & 0.9823 \\
\cline { 2 - 8 } & Val F1 & 0.9793 & 0.9817 & 0.9818 & 0.9818 & $\mathbf{0 . 9 8 3 2}$ & 0.9818 \\
\hline \hline \multirow{3}{*}{ ALBERT } & Train loss & 0.5384 & 0.2096 & 0.137 & 0.1061 & 0.0844 & 0.0682 \\
\cline { 2 - 8 } & Val Acc & 0.8909 & 0.9396 & 0.9438 & 0.9647 & 0.9616 & 0.9658 \\
\cline { 2 - 8 } & Val F1 & 0.8955 & 0.94 & 0.9445 & 0.9637 & 0.9607 & $\mathbf{0 . 9 6 5 1}$ \\
\hline
\end{tabular}

5 for DistilBERT, and 6 for ALBERT. Please refer to Table VIII to see the detail results of our validation experiments for choosing the number of epochs for each model.

\section{Results and Discussion}

In this section, we describe the experiment results for different experiment settings and test datasets. We compare our approach with different benchmark approaches and each of the benchmark approaches except NLM-BERT-17M are trained on the same training dataset that we describe in Section III-A.

Table IX displays the results of benchmark comparison. The results show that our transfer learning-based models outperform Hassanzadeh et al. [7]'s pretrained BERT and cosine similarity-based approach NLM-BERT model in terms of f1-score on at least 4 out of 5 test datasets. The NLMBERT-17M model has the best f1-scores for NATO-SFA, Risk Models, and CE Pairs. However, when the model is trained on our smaller $100 \mathrm{~K}$ causal pairs dataset with the original thresholds mentioned by the authors has zero true positive value on NATO-SFA and CE Pairs datasets. We get similar result on the Twitter dataset by using 0.94 and 0.90 as $t h_{1}$ and $t h_{2}$, respectively. NLM-BERT++ model outperforms the NLM-BERT model on all except the Risk Models dataset. In the Risk Models dataset, the f1-scores are comparable. Luo et al. [4]'s causal network based approach has varying results and its f1-scores and accuracy vary from 0.1590 to 0.6095 and from 0.49 to 0.5426 , respectively. Comparing among the BERT-based models, BERT has the best f1-scores on SemEval and Risk Models datasets, DistilBERT has the best f1-scores on NATO-SFA and CE Pairs datasets, while RoBERTa achieves the best f1-score on the Twitter dataset.

From these experiment results we observe that Hassanzadesh et al. [7]'s NLM-BERT model is dependent on large training dataset and expert supervision for manual threshold selection. Also, the thresholds are not transferable as they are test dataset specific. In this paper we show that we can solve this manual threshold selection problem by automatically learning thresholds from training data. The automatically selected thresholds are not dependent to any test data hence they are transferable. We also observe that the transfer learningbased approaches can achieve comparable performance to NLM-BERT-17M when fine-tuned on a small training dataset.
Although the training dataset is small the rich linguistic features captured by the pretrained transfer learning models enable them to achieve comparable performance. These results also demonstrate the effectiveness of our proposed training dataset preparation method where we automatically prepared a training dataset with $100 \mathrm{~K}$ causal pairs and the same number of non-causal pairs without any human annotation process.

\section{CONCLUSION}

In this paper, we have proposed a transfer learning-based approach to answer binary causal questions. We have presented a semi-supervised training dataset preparation approach which automatically collects the training data from news articles using a set of causal cue words. We have shown how to fine-tune a pretrained BERT model on our training dataset to answer binary casual questions. Our approach achieves a comparable performance to a number of benchmark approaches on five benchmark test datasets that are extracted by human experts. From experiments, we observe that we can solve the issue of large training dataset requirement of many machine learningbased models by fine-tuning a pretrained transfer learningbased model on a carefully designed small training dataset.

\section{REFERENCES}

[1] K. Radinsky, S. Davidovich, and S. Markovitch, "Learning causality for news events prediction," in $W W W$. ACM, 2012, pp. 909-918.

[2] M. Riaz and R. Girju, "Toward a better understanding of causality between verbal events: Extraction and analysis of the causal power of verb-verb associations," in SIGDIAL, 2013, pp. 21-30.

[3] — " "Recognizing causality in verb-noun pairs via noun and verb semantics," in EACL, 2014, pp. 48-57.

[4] Z. Luo, Y. Sha, K. Q. Zhu, S.-w. Hwang, and Z. Wang, "Commonsense Causal Reasoning between Short Texts," in KR, 2016, pp. 421-431.

[5] S. Sasaki, S. Takase, N. Inoue, N. Okazaki, and K. Inui, "Handling Multiword Expressions in Causality Estimation," IWCS, 2017.

[6] T. Dasgupta, R. Saha, L. Dey, and A. Naskar, "Automatic Extraction of Causal Relations from Text using Linguistically Informed Deep Neural Networks," in SIGDIAL, 2018, pp. 306-316.

[7] O. Hassanzadeh, D. Bhattacharjya, M. Feblowitz, K. Srinivas, M. Perrone, S. Sohrabi, and M. Katz, "Answering Binary Causal Questions Through Large-Scale Text Mining: An Evaluation Using Cause-Effect Pairs from Human Experts," in IJCAI, 2019.

[8] R. Sharp, M. Surdeanu, P. Jansen, P. Clark, and M. Hammond, "Creating causal embeddings for question answering with minimal supervision," in Conference on Empirical Methods in Natural Language Processing, Proceedings, 2016.

[9] H. Kayesh, M. Islam, and J. Wang, "On event causality detection in tweets," arXiv preprint arXiv:1901.03526, 2019. 
TABLE IX: Comparison with other benchmark methods (NR: not reported, NA: not applicable)

\begin{tabular}{|c|c|c|c|c|c|c|c|c|c|c|c|}
\hline Dataset & Method & \# of Pairs & th1 & th1 & tp & $\mathbf{f p}$ & Acc & Pre & Rec & F1 & AUC \\
\hline \multirow{8}{*}{ SemEval } & NLM-BERT-17M & $17 \mathrm{M}$ & 0.82 & 0.62 & 863 & 855 & 0.5050 & 0.5020 & 0.9980 & 0.6680 & NR \\
\hline & NLM-BERT & \multirow{7}{*}{$100 \mathrm{~K}$} & 0.82 & 0.62 & 225 & 63 & 0.5936 & 0.7812 & 0.2601 & 0.3903 & 0.5936 \\
\hline & NLM-BERT++ & & 0.60 & 0.30 & 865 & 860 & 0.5029 & 0.5014 & 1.0000 & 0.6680 & 0.5029 \\
\hline & CausalNet & & NA & NA & 79 & 50 & 0.5168 & 0.6124 & 0.0913 & 0.1590 & 0.5168 \\
\hline & BERT & & NA & NA & 862 & 816 & 0.5266 & 0.5137 & 0.9965 & 0.6779 & 0.5266 \\
\hline & RoBERTa & & NA & NA & 865 & 864 & 0.5006 & 0.5003 & 1.0000 & 0.6669 & 0.5006 \\
\hline & DistilBERT & & NA & NA & 864 & 855 & 0.5052 & 0.5026 & 0.9988 & 0.6687 & 0.5052 \\
\hline & ALBERT & & NA & NA & 854 & 810 & 0.5254 & 0.5132 & 0.9873 & 0.6754 & 0.5254 \\
\hline \multirow{8}{*}{ NATO-SFA } & NLM-BERT-17M & $17 \mathrm{M}$ & 0.94 & 0.82 & 58 & 53 & $\overline{0.5420}$ & 0.5230 & 0.9830 & $\overline{0.6820}$ & NR \\
\hline & NLM-BERT & \multirow{7}{*}{$100 \mathrm{~K}$} & 0.94 & 0.82 & 0 & 0 & 0.5000 & 0.0000 & 0.0000 & 0.0000 & 0.5000 \\
\hline & NLM-BERT++ & & 0.60 & 0.30 & 59 & 59 & 0.5000 & 0.5000 & 1.0000 & 0.6667 & 0.5000 \\
\hline & CausalNet & & NA & NA & 20 & 16 & 0.5339 & 0.5556 & 0.3390 & 0.4211 & 0.5339 \\
\hline & BERT & & NA & NA & 55 & 55 & 0.5000 & 0.5000 & 0.9322 & 0.6509 & 0.5000 \\
\hline & RoBERTa & & NA & NA & 56 & 57 & 0.4915 & 0.4956 & 0.9492 & 0.6512 & 0.4915 \\
\hline & DistilBERT & & $\mathrm{NA}$ & NA & 57 & 57 & 0.5000 & 0.5000 & 0.9661 & 0.6590 & 0.5000 \\
\hline & ALBERT & & NA & NA & 53 & 57 & 0.4661 & 0.4818 & 0.8983 & 0.6272 & 0.4661 \\
\hline \multirow{8}{*}{ Risk Models } & NLM-BERT-17M & $17 \mathrm{M}$ & 0.00 & 0.90 & 345 & 318 & 0.5370 & 0.5200 & 0.9380 & 0.6690 & NR \\
\hline & NLM-BERT & \multirow{7}{*}{$100 \mathrm{~K}$} & 0.00 & 0.90 & 402 & 402 & 0.5000 & 0.5000 & 1.0000 & 0.6667 & 0.5000 \\
\hline & NLM-BERT++ & & 0.60 & 0.30 & 402 & 402 & 0.5000 & 0.5000 & 1.0000 & 0.6667 & 0.5000 \\
\hline & CausalNet & & NA & NA & 320 & 328 & 0.4900 & 0.4938 & 0.7960 & 0.6095 & 0.4900 \\
\hline & BERT & & NA & NA & 398 & 396 & 0.5025 & 0.5013 & 0.9900 & 0.6656 & 0.5025 \\
\hline & RoBERTa & & NA & NA & 390 & 392 & 0.4975 & 0.4987 & 0.9701 & 0.6588 & 0.4975 \\
\hline & DistilBERT & & NA & NA & 389 & 393 & 0.4950 & 0.4974 & 0.9677 & 0.6571 & 0.4950 \\
\hline & ALBERT & & NA & NA & 387 & 388 & 0.4988 & 0.4994 & 0.9627 & 0.6576 & 0.4988 \\
\hline \multirow{8}{*}{ CE Pairs } & NLM-BERT-17M & $17 \mathrm{M}$ & 0.91 & 0.65 & 160 & 157 & 0.5090 & 0.5050 & 1.0000 & 0.6710 & NR \\
\hline & NLM-BERT & \multirow{7}{*}{$100 \mathrm{~K}$} & 0.91 & 0.65 & 0 & 0 & 0.5000 & 0.000 & 0.000 & 0.000 & 0.5000 \\
\hline & NLM-BERT++ & & 0.60 & 0.30 & 160 & 160 & 0.5000 & 0.5000 & 1.0000 & 0.6667 & 0.5000 \\
\hline & CausalNet & & NA & NA & 99 & 93 & 0.5188 & 0.5156 & 0.6188 & 0.5625 & 0.5187 \\
\hline & BERT & & NA & NA & 155 & 153 & 0.5062 & 0.5032 & 0.9688 & 0.6624 & 0.5062 \\
\hline & RoBERTa & & NA & NA & 150 & 158 & 0.4750 & 0.4870 & 0.9375 & 0.6410 & 0.4750 \\
\hline & DistilBERT & & NA & NA & 158 & 154 & 0.5125 & 0.5064 & 0.9875 & 0.6695 & 0.5125 \\
\hline & ALBERT & & NA & NA & 147 & 155 & 0.4750 & 0.4868 & 0.9187 & 0.6364 & 0.4750 \\
\hline \multirow{7}{*}{ Twitter } & NLM-BERT & \multirow{7}{*}{$100 \mathrm{~K}$} & 0.94 & 0.90 & $\overline{00}$ & $\overline{0}$ & 0.4989 & 0.000 & 0.000 & 0.000 & 0.5000 \\
\hline & NLM-BERT++ & & 0.60 & 0.30 & 459 & 457 & 0.5011 & 0.5011 & 1.0000 & 0.6676 & 0.5000 \\
\hline & CausalNet & & NA & NA & 232 & 192 & 0.5426 & 0.5472 & 0.5054 & 0.5255 & 0.5427 \\
\hline & BERT & & NA & NA & 444 & 439 & 0.5044 & 0.5028 & 0.9673 & 0.6617 & 0.5034 \\
\hline & RoBERTa & & NA & NA & 451 & 438 & 0.5131 & 0.5073 & 0.9826 & 0.6691 & 0.5121 \\
\hline & DistilBERT & & NA & NA & 442 & 436 & 0.5055 & 0.5034 & 0.9630 & 0.6612 & 0.5045 \\
\hline & ALBERT & & NA & NA & 409 & 395 & 0.5142 & 0.5087 & 0.8911 & 0.6477 & 0.5134 \\
\hline
\end{tabular}

[10] H. Kayesh, M. S. Islam, and J. Wang, "Event causality detection in tweets by context word extension and neural networks," in PDCAT, 2019, pp. 352-357.

[11] _ _ "A causality driven approach to adverse drug reactions detection in tweets," in ADMA, 2019, pp. 316-330.

[12] Y. Wu, M. Schuster, Z. Chen, Q. V. Le, M. Norouzi, W. Macherey, M. Krikun, Y. Cao, Q. Gao, K. Macherey, J. Klingner, A. Shah, M. Johnson, X. Liu, L. Kaiser, S. Gouws, Y. Kato, T. Kudo, H. Kazawa, K. Stevens, G. Kurian, N. Patil, W. Wang, C. Young, J. Smith, J. Riesa, A. Rudnick, O. Vinyals, G. Corrado, M. Hughes, and J. Dean, "Google's neural machine translation system: Bridging the gap between human and machine translation," CoRR, vol. abs/1609.08144, 2016.

[13] R. Girju, "Automatic detection of causal relations for question answering," in ACL Workshop on Multilingual Summarization and Question Answering, vol. 12, 2003, pp. 76-83.

[14] D. Corney, D. Albakour, M. Martinez, and S. Moussa, "What do a million news articles look like?" in NewsIR, 2016, pp. 42-47.

[15] J. Devlin, M.-W. Chang, K. Lee, and K. Toutanova, "Bert: Pre-training of deep bidirectional transformers for language understanding," arXiv preprint arXiv:1810.04805, 2018

[16] Y. Zhu, R. Kiros, R. Zemel, R. Salakhutdinov, R. Urtasun, A. Torralba, and S. Fidler, "Aligning books and movies: Towards story-like visual explanations by watching movies and reading books," in IEEE International Conference on Computer Vision, 2015, pp. 19-27.

[17] I. Hendrickx, S. N. Kim, Z. Kozareva, P. Nakov, D. Ó Séaghdha, S. Padó, M. Pennacchiotti, L. Romano, and S. Szpakowicz, "Semeval2010 task 8: Multi-way classification of semantic relations between pairs of nominals," in Workshop on Semantic Evaluations: Recent
Achievements and Future Directions, 2009, pp. 94-99.

[18] NATO, "Stratigic Foresight Analysis 2017 report," https://www.act.nato.int/publications-ffao, 2017, [Online; accessed February 21, 2019].

[19] S. Sohrabi, A. V. Riabov, M. Katz, and O. Udrea, "An ai planning solution to scenario generation for enterprise risk management," in Thirty-Second AAAI Conference on Artificial Intelligence, 2018.

[20] S. Sohrabi, M. Katz, O. Hassanzadeh, O. Udrea, M. D. Feblowitz, and A. Riabov, "Ibm scenario planning advisor: Plan recognition as ai planning in practice," AI Communications, no. Preprint, pp. 1-13, 2019.

[21] N. Reimers and I. Gurevych, "Sentence-bert: Sentence embeddings using siamese bert-networks," arXiv preprint arXiv:1908.10084, 2019.

[22] J. Johnson, M. Douze, and H. Jégou, "Billion-scale similarity search with gpus," arXiv preprint arXiv:1702.08734, 2017.

[23] T. Wolf, L. Debut, V. Sanh, J. Chaumond, C. Delangue, A. Moi, P. Cistac, T. Rault, R. Louf, M. Funtowicz, and J. Brew, "Huggingface's transformers: State-of-the-art natural language processing," ArXiv, vol. abs/1910.03771, 2019.

[24] Y. Liu, M. Ott, N. Goyal, J. Du, M. Joshi, D. Chen, O. Levy, M. Lewis, L. Zettlemoyer, and V. Stoyanov, "Roberta: A robustly optimized bert pretraining approach," arXiv preprint arXiv:1907.11692, 2019.

[25] V. Sanh, L. Debut, J. Chaumond, and T. Wolf, "Distilbert, a distilled version of bert: smaller, faster, cheaper and lighter," arXiv preprint arXiv:1910.01108, 2019.

[26] Z. Lan, M. Chen, S. Goodman, K. Gimpel, P. Sharma, and R. Soricut, "Albert: A lite bert for self-supervised learning of language representations," arXiv preprint arXiv:1909.11942, 2019. 